\title{
INTELLIGENT NAVIGATION DESIGN OF FULLY AUTONOMOUS UNMANNED AERIAL VEHICLE BASED ON LASER POSITIONING
}

\author{
Guanghua Nie \\ Henan Institute of Technology, Henan 453000, China \\ Email: nieguanghua453@163.com
}

\begin{abstract}
In order to study the intelligent navigation design of fully autonomous UAV (Unmanned Aerial Vehicle) for laser positioning, the laser positioning UAV is mainly discussed. An intelligent navigation design system of fully autonomous UAV based on laser positioning is adopted, which can be used not only for material measurement in large mines and wharfs, but also for mine safety and intelligent mining system. Compared with radar technology, acoustic detection and radio scanning, this method has the characteristics of high efficiency and easy realization, and is more suitable for the detection and positioning of low-speed flying small targets in ultra-low altitude visual range. Finally, the software of the system is programmed, including time, inclination, pendulum, positioning and other measurements. The experimental results are illustrated by flow chart. Laser positioning is designed separately according to the actual needs. The UAV and GPS (Global Position System) positioning sensor system are studied and analysed, and the two are combined to get three-dimensional coordinates. The test results show that the system can basically fulfill the requirement of autonomous range of laser positioning, and provide data support for the later positioning system.
\end{abstract}

Keywords: Laser Positioning, UAV, Navigation.

\section{Introduction}

The application of automation in aircraft piloting is a great progress in science and technology. With the rapid development of microelectronics, microelectromechanical technology, information technology, intelligent technology and aviation technology, UAV (Unmanned Aerial Vehicle) has developed rapidly. It uses wireless remote control or embedded program control instead of manual work to accomplish various tasks. With its advantages of small size, low cost, good concealment, convenient use, low requirement for flight environment and strong survivability, UAV has been widely used in military and civil fields. In several local wars, UAVs have played an important role in reconnaissance, attack and electronic countermeasures. In future wars, UAVs will have greater significance.

Military countries around the world have stepped up the development of UAVs. In the civil field, UAV is widely used in aerial photography, disaster monitoring, communication relay, meteorological detection, traffic patrol and pesticide spraying.

UAV navigation system is one of the key components of UAV system. At present, the navigation technologies used by UAV mainly include inertial navigation, satellite navigation, Doppler navigation, visual navigation, terrain assistance, and geomagnetic field navigation.
In practical application, it is necessary to select the appropriate navigation system according to the UAV's flight environment, mission, application scope, and conditions of navigation technology.

\section{Literature Review}

Laser positioning system has been explored by Guerra et al. [1] for a long time, Gallay et al. [2] gradually improved the maturity of photoelectric technology and $\mathrm{Lu}$ et al. [3] further discussed the electronic technology. Laser rangefinder products have gone through three generations, and have been updated to the third generation.

The main characteristics of the first two generations of products include large volume, high power consumption, large error, high cost, single function etc. Sun et al. [4] pointed out that, at present, laser positioning is mainly developing towards the direction of miniaturization, low power consumption, high precision, low cost, and multifunction.

Liu et al. [5] stated that, after the appearance of the photoelectric ranging sensor system based on laser as carrier, because of its excellent performance, has been rapidly applied to the national economy and industrial development, such as geodetic survey, aerospace survey, indoor positioning, UAV and other fields. 
The invention of laser marks a new level of human use and mastery of light. Mac et al. [6] thought that, compared with electromagnetic wave, laser has good physical properties, such as directivity, mono chromaticity and coherence. Bu et al. [7] proposed that the main characteristics of laser are pulse characteristics output stability etc. He et al. [8] believed that laser ranging sensor system is an instrument which measures distance depending on the unique properties of laser such as good collimation and small divergence angle. Because of the special performance of laser, the laser ranging system has a unique style and laid a foundation for its development in various fields.

Vetrella et al. [9] considered that the greatest advantage of laser ranging sensor system is indirect measurement. First, laser pulses (single pulse or multiple pulses) are emitted by the laser, which are reflected back after reaching the target. By calculating the time or the total phase difference, the distance can be obtained indirectly. The measurement method is simple and safe, so it has been widely used.

In this technology field, Europe and the United States remain in the lead. Piotr et al. [10] pointed out that Europe mainly focuses on hand-held laser positioned, while the United States focuses on military ranging. Nguyen et al. [11] held that the research on laser positioning in China began in the mid-1980s, and started late. It was developed on the basis of a laser generator with solid and gas medium.

Although the research on laser positioning in China is progressing and improving, there is still a lack of high-performance products, and most of the products on the market are phase laser positioners. Jin et al. [12] pointed out that although this kind of positioning has high accuracy, it has short range, poor stability and weak anti-interference ability. However, positioning systems with high accuracy, small size and portability, are not widely used in the market due to high cost and power consumption.

Therefore, it is still necessary to continue to improve, to progress, to develop better performance, lighter quality, lower power consumption and lower cost products, to serve the public, and to meet their needs.

In order to intercept low-attitude, slow-speed, small flight target (abbreviated as low, slow, and small target) effectively, it is necessary to first realize the track measurement and positioning of small and slow targets. A series of research on track measurement and positioning of low-altitude and slow-speed small target in urban environment is carried out, and a set of track measurement and positioning system for low, slow, and small target in urban environment is designed.

The working environment of the system is complex electromagnetic, complex meteorology, and noisy urban areas, and the particularity of low-speed and small targets, the fuselage of the system is mostly made of non-metallic materials, the radar reflection area is small, and the Doppler effect caused by low, slow and small target is not obvious, so the effect of traditional radar detection methods on detecting slow and small targets in urban environment is poor. Because of the interference of ambient noise and the low noise processing of low, slow, and small target motor, the efficiency of acoustic detection is low. Due to the existence of various electromagnetic interference, the detection effect of radio detection for low, slow, and small targets is not ideal. In contrast, laser detection can use the characteristics of short duration and high instantaneous power of laser pulse to measure the slant distance, height, velocity and pitch angle of low and slow small targets, so as to locate them.

The purpose is to use laser detection technology to realize track measurement and location of low, slow, and small target, overcome the shortcomings of traditional radar for low, slow, and small target detection, and provide preconditions for intercepting low, slow, and small target. With the development of science and technology, the functions of low, slow and small targets, especially those represented by low-complexity models, have become more powerful and the cost has gradually decreased. The application prospects of low, slow, and small target will gradually expand, and it will bring more and more security problems. It will influence air routes, divulge State secrets, expose personal privacy and even be used by terrorists, which may cause serious harm to national security and people's lives and property.

Therefore, the research on the detection and interception technology of low, slow, and small targets has a strong practical significance.

\section{Principle and Test of Detection and Positioning System}

\subsection{Measuring principle}

Navigation algorithm can give the attitude, velocity and position information of the carrier, which is the most important prerequisite for UAV to achieve autonomous control. At present, the main navigation systems used for UAV flight control at home and abroad include: INS (Inertial Navigation System), Gravity Field Complementary Filtering Navigation System, GPS (Global Positioning System) and GPS/INS integrated navigation system. As the feature extraction algorithm of positioning in navigation system, they should have the following characteristics:

Stability: For each observed feature point, the feature extraction algorithm should be able to extract features independent of distance and angle. When these feature points are observed again, they should be accurately recognized again by feature 
extraction algorithm. This ensures that features can be effectively used in subsequent data processing.

Efficiency: As an integral part of navigation system, feature extraction algorithm should be able to execute in real time. Offline navigation system is not practical for UAV. This means that the algorithm should consume less computing resources and have higher execution efficiency.

Robustness: Noise pollution inevitably exists in the acquisition process of sensor data, and noise easily interferes with the accurate feature extraction by feature detectors. Therefore, feature extraction method should have high anti-jamming ability, restrain the influence of noise on feature detection, and reduce the false detection rate of features as much as possible.

Distinguish ability: In order to minimize the impact of environmental similarity on distinguishing feature points, feature extraction algorithm should give each feature point some unique features to distinguish from other feature points. The unique feature information can correct the wrong filtering results to the greatest extent. Therefore, feature extraction algorithm should be able to capture the characteristics of the environment, and use these characteristics to support subsequent data association algorithms.

A vertical downward airborne camera is used to obtain image information and extract feature points. Based on the above principles, corner detection method is used to extract feature points in images, which is commonly used and relatively mature in the field of image processing. In order to extract features from the pyramids of images, and to extract features from obstacles with little feature information and obstacles with too much feature information, a multi-scale Kanade-Tomasi corner detector is used.

The detector can extract corners in different scales. The extracted features need to be described by feature descriptor, so as to provide more information for data association and further improve the robustness of data association. SIFT (Scale Invariant Feature Transform) descriptor is used to describe features, which has the advantages of scale invariance and rotation invariance.

Distance measurement is achieved by calculating the distance of laser propagation in the time interval between the transmitted signal and the echo signal, as shown in formulas (1), (2), and (3):

$$
\begin{aligned}
& 1_{1}=\frac{1}{2 n} c_{0} * \Delta t_{1} \\
& 1_{2}=\frac{1}{2 n} c_{0} * \Delta t_{2} \\
& 1_{3}=\frac{1}{2 n} c_{0} * \Delta t_{3}
\end{aligned}
$$

Among them: $I_{1}, I_{2}$, and $I_{3}$ refer to the linear distance between the detection origin 0 and the position points of $\mathrm{P}_{1}, \mathrm{P}_{2}$, and $\mathrm{P}_{3} ; \mathrm{n}$ is the average refractive index of the transmission medium to the laser under standard atmospheric conditions; $\mathrm{C}_{\mathrm{O}}$ represents the velocity of vacuum light; $\Delta \mathrm{t}_{1}$ and $\Delta t_{2}$ are the time for the laser pulse to refract back to the 0 point after it reaches the position $\mathrm{P}_{1}, \mathrm{P}_{2}$, and $\mathrm{P}_{3}$ from the detection origin 0 , respectively.

On the basis of completing distance measurement, the altitude information of low, slow, and small target is obtained by measuring the inclination of the detection device and further processing, as shown in formulas (4), (5), and (6):

$$
\begin{aligned}
& \mathrm{h}_{1}=1_{1} \sin \beta_{1} \\
& \mathrm{~h}_{2}=1_{2} \sin \beta_{2} \\
& \mathrm{~h}_{3}=1_{3} \sin \beta_{3}
\end{aligned}
$$

In the above formulas, $\mathrm{h}_{1}, \mathrm{~h}_{2}$, and $\mathrm{h}_{3}$ indicate the horizontal height between the position points $\mathrm{P}_{1}, \mathrm{P}_{2}$, and $\mathrm{P}_{3}$ and the ground, respectively; $\beta_{1}, \beta_{2}$, and $\beta_{3}$ are the angle between the axial direction of the detector and the horizontal plane when measuring three times.

After the distance and height data are obtained, the azimuth angle of the detection device is further measured, and the position coordinate information of low, slow, and small target is obtained by further calculation and processing, as shown in formulas (7), (8), and (9):

$$
\begin{aligned}
& \mathrm{P}_{1}\left(\mathrm{x}_{1}, \mathrm{y}_{1}, \mathrm{z}_{1}\right):\left\{\begin{array}{l}
\mathrm{x}_{1}=1_{2} \cos \beta_{2} \cos \left(\pi-\gamma_{1}\right) \\
\mathrm{y}_{1}=1_{1} \cos \beta_{1} \sin \left(\pi-\gamma_{1}\right) \\
\mathrm{z}_{1}=1_{1} \sin \beta_{1}
\end{array}\right. \\
& \mathrm{P}_{2}\left(\mathrm{x}_{2}, \mathrm{y}_{2}, \mathrm{z}_{2}\right):\left\{\begin{array}{l}
\mathrm{x}_{2}=1_{2} \cos \beta_{2} \cos \gamma_{2} \\
\mathrm{y}_{2}=1_{2} \cos \beta_{2} \sin \gamma_{2} \\
\mathrm{z}_{2}=1_{2} \sin \beta_{2}
\end{array}\right. \\
& \mathrm{P}_{3}\left(\mathrm{x}_{3}, \mathrm{y}_{3}, \mathrm{z}_{3}\right):\left\{\begin{array}{l}
\mathrm{x}_{3}=1_{3} \cos \beta_{3} \cos \gamma_{3} \\
\mathrm{y}_{3}=1_{3} \cos \beta_{3} \sin \gamma_{3} \\
\mathrm{z}_{3}=1_{3} \sin \beta_{3}
\end{array}\right.
\end{aligned}
$$

In the formula, $\mathrm{P}_{1}\left(\mathrm{x}_{1}, \mathrm{y}_{1}, \mathrm{z}_{1}\right), \mathrm{P}_{2}\left(\mathrm{x}_{2}, \mathrm{y}_{2}, \mathrm{z}_{2}\right)$, and $\mathrm{P}_{3}\left(\mathrm{x}_{3}, \mathrm{y}_{3}, \mathrm{z}_{3}\right)$ are the coordinates of the position points $P_{1}, P_{2}$, and $P_{3} ; \gamma_{1}, \gamma_{2}$, and $\gamma_{3}$ are the angle between the axis direction of the overhead view (i.e. the north direction of the geomagnetic field) of the detection device rotating clockwise with the $\mathrm{O}_{\mathrm{x}}$ axis.

Velocity measurement includes the total velocity of the target and the velocity components along the $\mathrm{O}_{\mathrm{x}}$ axis, $\mathrm{O}_{\mathrm{y}}$ axis and $\mathrm{O}_{\mathrm{z}}$ axis. In triangle $\mathrm{OP}_{1} \mathrm{P}_{2}$ and triangle $\mathrm{OP}_{2} \mathrm{P}_{3}$, according to the cosine theorem, there are formulas (10) and (11): 
$1_{12}=\sqrt{1_{1}^{2}+1_{1}^{2}-21_{1} 1_{2} \cos \theta_{1}}$

$1_{23}=\sqrt{1_{2}^{2}+1_{3}^{2}-21_{2} 1_{3} \cos \theta_{2}}$

$\theta_{1}$ and $\theta_{2}$ are the pendulum angle of the detection device with 0 as the origin. Because of the short turning time $\Delta \mathrm{t}_{1}$ and $\Delta \mathrm{t}_{2}$ of the pendulum angles $\theta_{1}$ and $\theta_{2}$ of detection device, the average velocity of the target passing through $\mathrm{P}_{1} \mathrm{P}_{2}$ and $\mathrm{P}_{2} \mathrm{P}_{3}$ is approximately equal to the instantaneous velocity of the target, as shown in formulas (12) and (13):

$$
\begin{aligned}
& \mathrm{v}_{12}=\frac{\mathrm{l}_{12}}{\Delta \mathrm{t}_{1}} \\
& \mathrm{v}_{23}=\frac{\mathrm{l}_{23}}{\Delta \mathrm{t}_{2}}
\end{aligned}
$$

The mean values of $\mathrm{v}_{12}$ and $\mathrm{v}_{23}$ are solved, and the approximate velocity of small target is obtained shown in Formula (14):

$$
\mathrm{v}=\frac{\mathrm{v}_{12}+\mathrm{v}_{23}}{2}
$$

In order to further predict the velocity of small target at the next moment, it is necessary to measure the velocity components $\mathrm{v}_{\mathrm{x}}, \mathrm{v}_{\mathrm{y}}$, and $\mathrm{v}_{\mathrm{z}}$ of $\mathrm{O}_{\mathrm{x}}$ axis, $\mathrm{O}_{\mathrm{y}}$ axis and $\mathrm{O}_{\mathrm{z}}$ axis direction, as shown in formulas (15) and (16):

$$
\begin{aligned}
& \mathrm{v}_{\mathrm{x} 12}=\frac{\mathrm{dx}_{12}}{\mathrm{dt}}=\frac{\Delta \mathrm{x}_{12}}{\Delta \mathrm{t}_{1}}=\frac{\mathrm{x}_{2}-\mathrm{x}_{1}}{\Delta \mathrm{t}_{1}} \\
& \mathrm{v}_{\mathrm{x} 23}=\frac{\mathrm{dx}_{23}}{\mathrm{dt}}=\frac{\Delta \mathrm{x}_{23}}{\Delta \mathrm{t}_{2}}=\frac{\mathrm{x}_{3}-\mathrm{x}_{2}}{\Delta \mathrm{t}_{2}}
\end{aligned}
$$

In the above formula, $\mathrm{V}_{\mathrm{x} 12}$ and $\mathrm{V}_{\mathrm{x} 23}$ are the mean velocity between $\mathrm{P}_{1} \mathrm{P}_{2}$ and $\mathrm{P}_{2} \mathrm{P}_{3}, \Delta \mathrm{t}_{1}$ and $\Delta \mathrm{t}_{2}$ are the time interval between two adjacent measurements. Because $\Delta \mathrm{t}_{1}$ and $\Delta \mathrm{t}_{2}$ are short, the velocity along the $\mathrm{O}_{\mathrm{x}}$ axis of small target can be obtained by taking the average value of $\mathrm{V}_{\mathrm{x} 12}$ and $\mathrm{V}_{\mathrm{x} 23}$ of the target through $\mathrm{P}_{1}, \mathrm{P}_{2}$, and $\mathrm{P}_{3}$.

$\mathrm{v}_{\mathrm{x}}=\frac{\mathrm{v}_{\mathrm{x} 12}+\mathrm{v}_{\mathrm{x} 23}}{2}$

Similarly, the velocity of the small target along the $O_{y}$ axis and along the $O_{z}$ axis is obtained as shown in formulas (17), (18), and (19):

$$
\begin{aligned}
& \mathrm{v}_{\mathrm{y}}=\frac{\mathrm{v}_{\mathrm{y} 12}+\mathrm{v}_{\mathrm{y} 23}}{2} \\
& \mathrm{v}_{\mathrm{z}}=\frac{\mathrm{v}_{\mathrm{z} 12}+\mathrm{v}_{\mathrm{z} 23}}{2}
\end{aligned}
$$

After the velocity data are obtained, the acceleration data are obtained by deriving the velocity data, as shown in formulas (20), (21), and (22):

$$
\begin{aligned}
& \mathrm{a}_{\mathrm{x}}=\frac{\mathrm{dv}_{\mathrm{x}}}{\mathrm{dt}}=\frac{\Delta \mathrm{v}_{\mathrm{x}}}{\Delta \mathrm{t}_{1}}=\frac{\mathrm{v}_{\mathrm{x} 23}-\mathrm{v}_{\mathrm{x} 12}}{\Delta \mathrm{t}_{1}} \\
& \mathrm{a}_{\mathrm{y}}=\frac{\mathrm{dv} \mathrm{y}_{\mathrm{y}}}{\mathrm{dt}}=\frac{\Delta \mathrm{v}_{\mathrm{y}}}{\Delta \mathrm{t}_{1}}=\frac{\mathrm{v}_{\mathrm{y} 23}-\mathrm{v}_{\mathrm{y} 12}}{\Delta \mathrm{t}_{1}} \\
& \mathrm{a}_{\mathrm{z}}=\frac{\mathrm{dv_{ \textrm {z } }}}{\mathrm{dt}}=\frac{\Delta \mathrm{v}_{\mathrm{z}}}{\Delta \mathrm{t}_{1}}=\frac{\mathrm{v}_{\mathrm{z} 23}-\mathrm{v}_{\mathrm{z} 12}}{\Delta \mathrm{t}_{1}}
\end{aligned}
$$

In the formula: $a_{x}$ refers to the acceleration of small target along $\mathrm{x}$ direction, $\mathrm{a}_{\mathrm{y}}$ suggests the acceleration of small target along y direction, and $\mathrm{a}_{\mathrm{z}}$ indicates the acceleration of small target along $\mathrm{z}$ direction.

\subsection{Summary of laser positioning principle}

Track measurement and positioning of low, slow, and small target is based on laser ranging, so the principle of laser ranging is briefly summarized. At present, the common ranging methods mainly include ultrasonic ranging, radio frequency electromagnetic wave ranging, and laser ranging.

Compared with other methods, laser ranging has the advantages of strong anti-interference ability, long measurement distance, and high accuracy.

There are two main ways to realize laser ranging.

One is phase laser ranging, which indirectly measures the laser round trip time by measuring the phase change of the laser signal propagating in the range of measurement. The second is pulse laser ranging, which directly measures the time interval between the laser pulse transmitting signal and the echo signal.

The principle of phase laser ranging is to indirectly measure the laser round trip time $t$ by measuring the phase change of the laser signal propagating in the measurement range of 1 . The phase of light will change continuously when it propagates. When the propagation distance of light signal is wavelength of $\lambda$, the phase change is $2 \pi$.

As a result, when the measurement distance is 1 , assuming the phase difference between the laser emission signal and the echo signal is $\varphi$, the relationship between the measurement distance 1 , phase difference $\varphi$ and wavelength $\lambda$ is: $21=\lambda \times \varphi /(2 \pi)$ or $1=\lambda \times \varphi /(4 \pi)$.

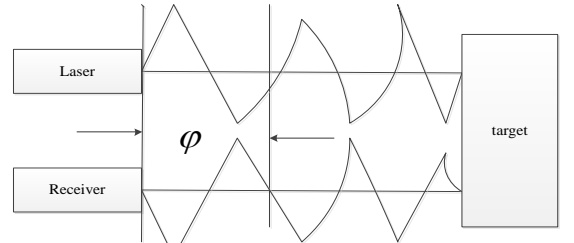

Figure 1: Principle diagram of phase laser ranging and positioning 
Define $s=\lambda / 2$ as the length of the ruler.

If $\varphi=2 \pi \mathrm{N}+\Delta \varphi$, where $\mathrm{N}$ is a natural number, $\Delta \varphi$ is the end in which $\varphi$ is less than $2 \pi$, and taking into account $s=\lambda / 2,1$ can be expressed as Formula (23):

$$
1=\mathrm{s}[\mathrm{N}+\Delta \varphi / 2 \pi]
$$

However, the positive period number $\mathrm{N}$ of laser signal cannot be determined in the measurement process, and only the phase shift $\Delta \varphi$ less than $2 \pi$ can be determined, so it is impossible to measure accurately.

Therefore, when the measuring distance 1 is larger than the length $s$ of the ruler, it is impossible to use only one light wave ruler for phase laser ranging. Only when the measuring distance 1 is less than the length of the gauge $s, 1$ can be determined and measured, as shown in Formula (24):

$1=s \times \Delta \varphi / 2 \pi$

From the above analysis, it can be seen that for long-distance measurement, it is necessary to choose a lower frequency gauge to ensure that the measuring distance 1 is larger than the length $s$ of the gauge, only in this way can the value of 1 be determined.

However, due to the phase measurement error which cannot be eliminated in the measuring equipment, the increase of s will cause / increase the measurement error and reduce the ranging accuracy. In order to solve this problem, a variety of length gauges can be selected to be used in a single measurement, taking into account the measurement distance and accuracy.

But this will inevitably increase the complexity of the system structure and reduce the ranging speed.

Pulse ranging method is based on laser Qswitching technology, which compresses the laser energy into a very narrow pulse to emit, so as to improve the peak power of the light source.

Therefore, the pulse ranging method is more suitable for target detection and tracking, especially for the measurement of uncooperative targets.

Set 1 to be the distance to be measured, $t$ is the time of flight of laser pulse, and $c_{o}$ is the speed of propagation of light.

Ignoring the influence of air tribute, humidity, temperature and other factors, the distance to be measured is shown in Formula (25):

$$
1=\mathrm{c}_{\mathrm{o}} \times \mathrm{t} / 2
$$

The measurement principle of pulse laser ranging method is shown in Figure 2.

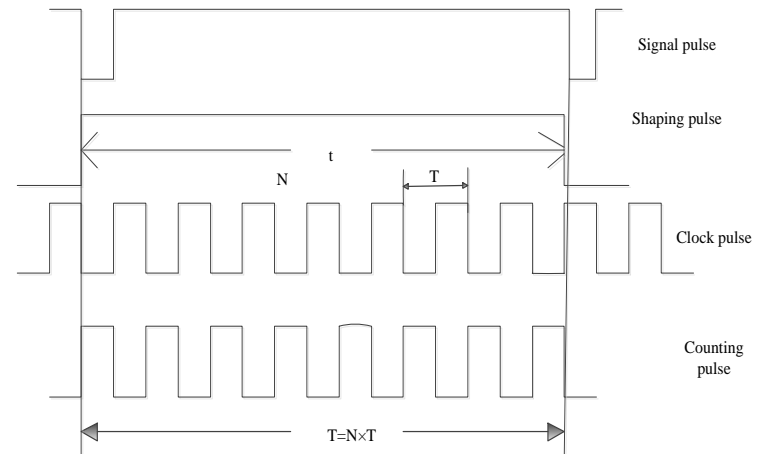

Figure 2: Pulsed laser ranging positioning principle

Within the time of $t$, the number of clock pulses of the counter is $\mathrm{N}$, the clock pulse frequency is $\mathrm{f}_{\mathrm{o}}$, and the distance to be measured is shown in Formula (26):

$1=\frac{\mathrm{c}_{\mathrm{o}}}{2} \mathrm{NT}=\frac{\mathrm{C}_{\mathrm{O}}}{2 \mathrm{f}_{\mathrm{o}}} \mathrm{N}=1_{\mathrm{o}} \mathrm{N}$

In Formula (26), $1_{\mathrm{o}}=\mathrm{c}_{\mathrm{o}} / 2 \mathrm{f}_{\mathrm{o}}$ represents the distance increment corresponding to clock pulse.

Obviously, the pulse laser ranging accuracy is inversely proportional to the clock pulse frequency $\mathrm{f}_{\mathrm{o}}$.

Through the analysis of two kinds of measurement method of measuring principle in phase laser ranging method, pulsed laser ranging method has many advantages:

Pulse laser ranging method has strong antiinterference and high ranging reliability when the total output optical power is the same. Because pulsed laser can output instantaneous power with higher intensity, the echo signal with higher intensity can be obtained. Reduce the influence of stray light, haze and other factors in the external environment, reduce the false trigger rate of the system, and improve the reliability.

It has faster measurement speed. As long as the received echo signal, pulse laser rangefinder can finish measurement, while the laser pulse flight time can be measured, the distance to be measured is calculated, and the process time required is very short. Phase laser ranging method requires calculating the phase difference between two adjacent pulses, and indirectly calculating the flight time pulse, so the measurement process requires for a relatively long time.

There is no need for mirrors and other matching devices to measure. Phase laser ranging method needs to measure the phase difference between continuous laser pulses. In order to improve the echo signal power, it is usually necessary to place mirrors and other matching devices at the target. 
Pulse laser ranging can output high-intensity instantaneous power, and the echo signal can be effectively detected by photo detectors, without the need for mirrors and other coordination devices.

This is very important for some special applications. In a large gathering places under the environment of city, key security area and the airport security area, the time for low, slow, and small target from discovery to the disposal is very short, so it needs as soon as possible to complete the track measurement and positioning of small target.

But low, slow, and small target, because of the small volume, the laser reflection area is small, so the light wave signal reflected is relatively weak, and it is necessary to improve the output power of the laser.

Considering these characteristics, the pulse laser ranging method is more suitable for the realization of low, slow, and small target location.

\subsection{Design of time interval measuring program}

The normal operation of the system in addition to a reasonable hardware circuit, the software must also be combined with high efficiency.

The software program can greatly improve the efficiency of the system and improve the detection accuracy of the system.

The software program that the system needs to design includes the time interval measurement procedures, inclination angle measurement procedures, pendulum angle measurement procedures, and azimuth measurement procedures.

The system selects time interval measurement chip TDC-GP21 to realize the laser pulse echo signal measurement and time interval, needs to make initialization, control measurement, data processing, and data reading of TDC-GP21 through the STM32F407.

TDC-GP21 initialization configuration includes measurement range selection, selection of the reference clock, the number of sampling set required, the calibration selection setting, definition of ALU data processing algorithm, selection of the input trigger mode, and opening interruption.

After initialization, the TDC-GP21 high-speed measurement unit receives the start signal and starts to work, reaching the set sampling number or encountering measurement overflow, triggering interruption, judging whether the measurement is normal or not. If it is normal, read data; otherwise, re-measure. STM32F407 and TDC-GP21 communicate through SPI (Single Program Initiation) interface. The core program of time interval measurement mainly includes TDC-GP21 initialization program and measurement program.

After reading the data from TDC-GP21, STM32F407 further processes the data and calculates the distance between the small targets.
After initializing TDC-GP21 in the measurement process, only after receiving the start signal, the measurement will be triggered.

Then, no matter whether it is measured normally or the stop echo signal is not received within the specified time, the pin of TDC-GP21 will have interrupt signal, and the content of the state register will be judged to determine whether the measurement is normal.

Before measurement triggering, the pins EN_START and EN_STOP need to be set high to enable start and stop channels, as shown in Figure 3.

The system uses the digital gyro L3G4200D for the realization of angle measurement.

Through STM32F407, the initialization configuration, control measurement, data processing and data reading of L3G4200D are carried out.

L3G4200D initialization configuration mainly includes the output data rate, data acquisition rate, and power supply mode, enables the measurement axis, the internal high pass filter mode and cut-off frequency setting, and makes the DRDY interrupted.

After the initial configuration, opening DADY interrupt, L3G4200D enters the measuring state, waits for interrupt, and after interruption triggering, calculates the swing angle of register data. STM32F407 and L3G4200D communicate through the IPC interface, core angle measurement including STM32F407 and L3G4200D communication program, and L3G4200D initialization procedure.

Read the L3G4200D internal angular velocity data, as shown in Figure 4:

The system chooses three-axis magnetometer and accelerometer integrated LSM303DLH to realize the azimuth measurement.

Through STM32F407, initialization configuration, control measurement, data processing and data reading of LSM303DLH are carried out.

LSM303DLH initialization configuration includes two parts, one is the accelerometer configuration initialization, including setting the measurement range, data output number setting, measurement mode, data acquisition rate etc. the other is the initial configuration of magnetometer, which is mainly measurement mode selection.

After the initial configuration is completed, opening DADY interrupt, LSM303DLH enters the measuring state and waits for interrupt, and after interrupt processing, calculates the azimuth read data register.

STM32F407 and LSM303DLH communicate through the Ic interface, core azimuth measurement includes STM32F40755 and LSM303DLH communication program, LSM303DLH initialization procedure, and reading the LSM303DLH internal magnetic field intensity and acceleration data etc.

Deflection angle measurement process is shown in Figure 5: 


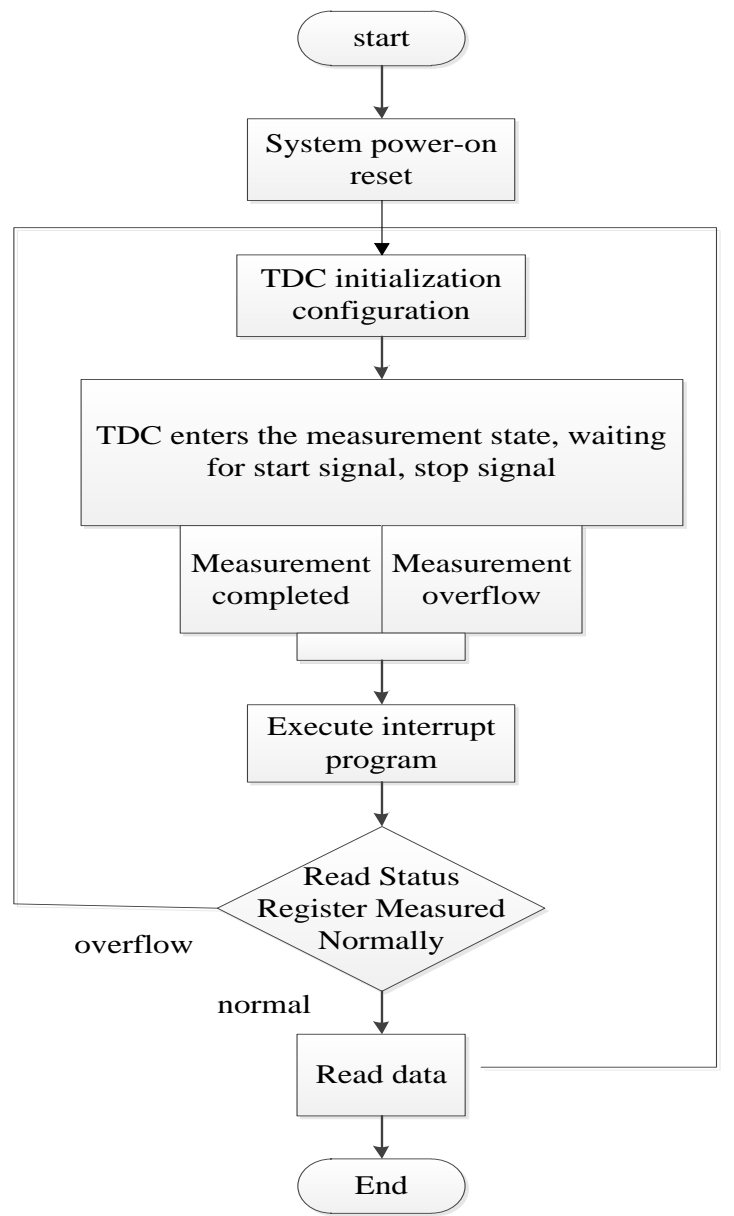

Figure 3: Flow chart of inclination measurement

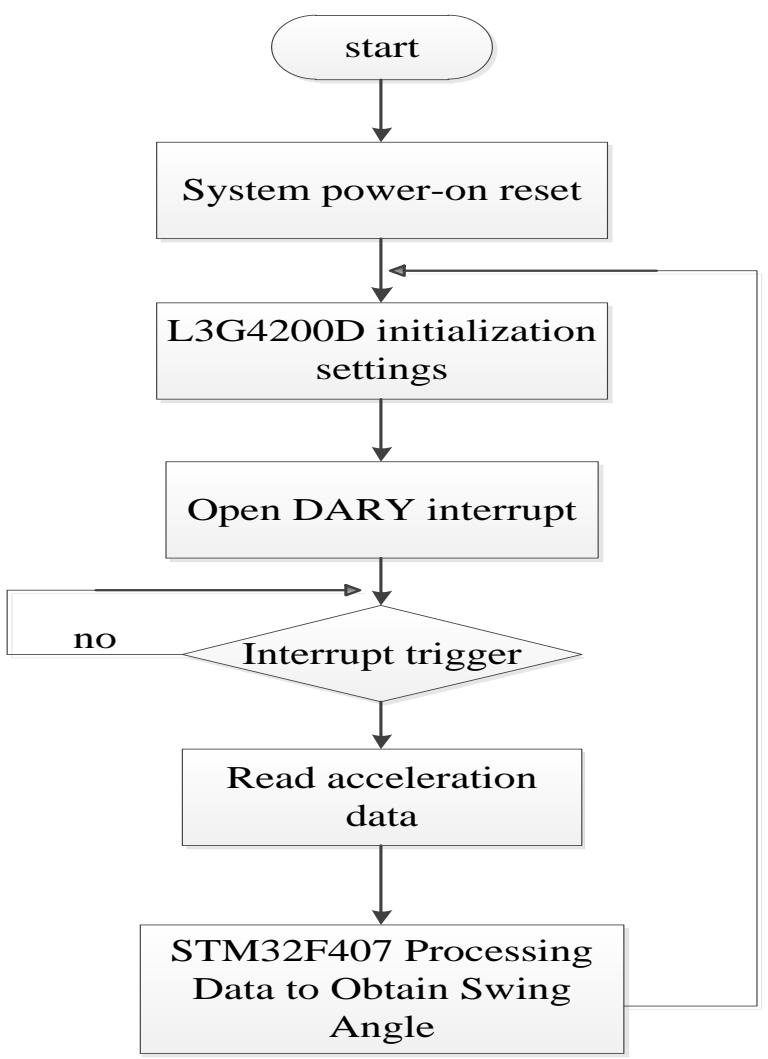

Figure 4: Flow chart of pendulum angle measurement

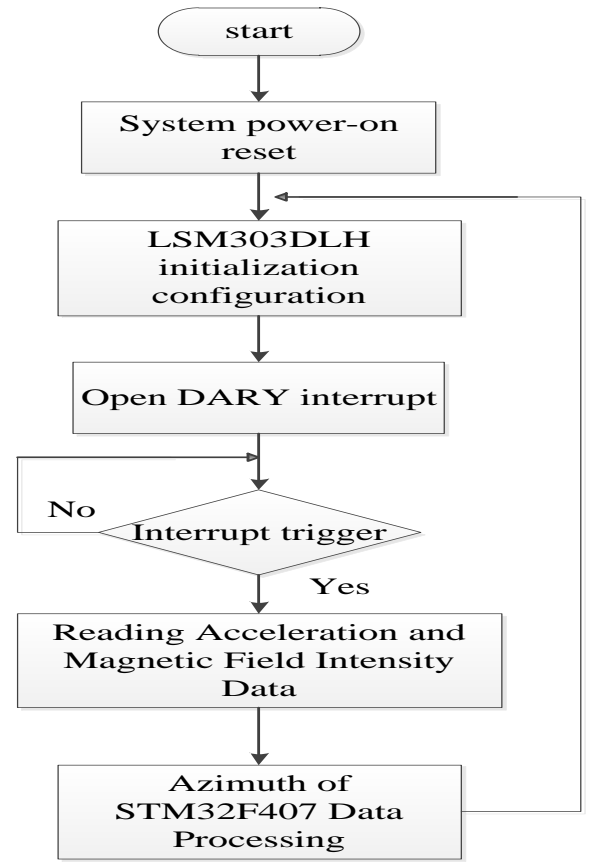

Figure 5: Flow chart of azimuth measurement

The following two parts of work have been completed:

The software program of the system is designed, including time interval measurement program, inclination measurement program, pendulum angle measurement program, and azimuth measurement program. The design idea of the program is explained in detail in the way of flow chart.

The test results of track measurement and positioning system for typical low, slow, and small targets are obtained, and the results are analyzed. The measurement of distance, height, velocity and coordinate position of small target is completed, and the error analysis of the test results is carried out.

The test results show that the system can basically detect and locate low, slow, and small targets, and the test error is within the range of system requirements, which provides effective data support for trajectory calculation and delay design in the follow-up study of low, slow, and small targets interception.

\subsection{Positioning and structure design of UAV}

The hardware platform used here is ARDrone, a small commercial Four-rotor UAV of Parrot Company. Compared with other popular Four-rotor UAVs, such as the Pelican and Hummingbird Fourrotor UAVs of Ascending Technology, Germany, the UAV has the advantages of low cost, light weight, strong structure and reliable protection design.

A standard ARDrone UAV is mainly composed of a main control board, navigation board, camera, WIFI module, electrical controller, etc. The navigation board is connected with sonar, gyroscope, accelerometer, barometer, geomagnetometer and other sensors, and is responsible for reading these 
sensor data and sending them to the main control board. The main control board is connected with navigation board, camera, WIFI module, electrical controller, etc., and is responsible for ARDrone overall control. Its structure is shown in Figure 6.

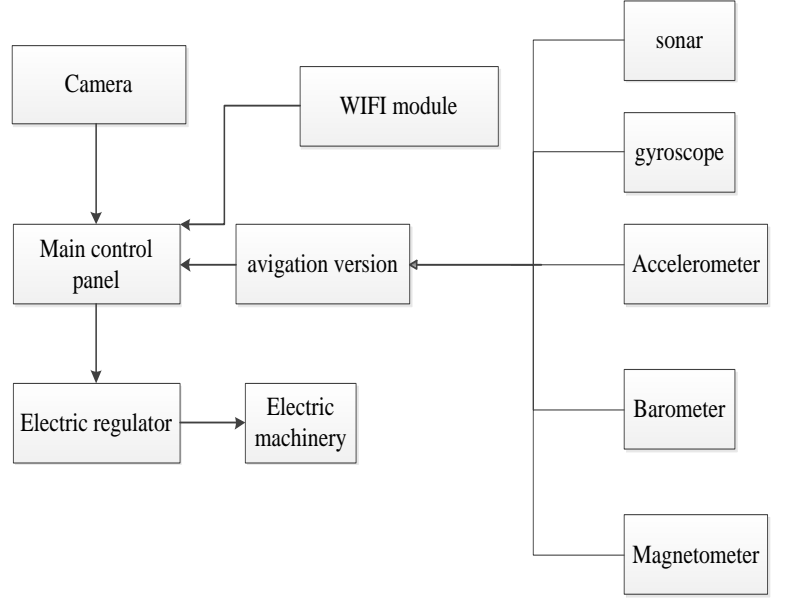

Figure 6: ARDrone hardware structure diagram

The positioning system designed is divided into two parts. The first part is a positioning method based on MU and optical flow technology. This method has little impact on the environment and high location accuracy in a short time, but it is easy to diverge for a long time, which leads to the failure of location. The second part is a location method based on feature matching. This method has high requirements on the environment, a large amount of calculation, but the location is more accurate, and there is no divergence problem. The idea is to use the second method to assist the first method, so as to alleviate the problem of positioning divergence of the latter.

Because the update frequency of MU and control data stream is the fastest, the positioning system acquires IMU and control data and then estimates the current motion state in real time according to the pre-established UAV kinematics model. Considering that the calculation of optical flow needs a lot of computing time, the program to implement the algorithm is placed in a single thread. Every time an image arrives, the image is placed in the buffer. If the buffer has an old image, it will be covered. When the thread has processed one frame of image, it reads the next frame from the buffer. The purpose of this method is to ensure that all the images processed by optical process are up-to-date and discard the old images directly when the computing resources are insufficient.

The velocity information obtained from the optical flow is sent to the state estimator for processing as observation. It is noted that all data transmission and processing have time delays, and the real time points of these data need to be taken into account when sending them to the state estimator.
The positioning system can only maintain accurate positioning in a short time when it only depends on IMU and optical flow. In order to make the system work for a long time, every certain time, make the UAV into hovering state; in the case of maintaining the stability of the body, the positioning system obtains the image data of the vertical downward camera, and the feature matching method mentioned earlier finds the optimal matching in the global map obtained in advance, so as to get the position of the UAV in the global coordinate system.

The position is fed into the state estimator with high confidence and maintained for a period of time, so that the system can converge the estimated position to the vicinity of the coordinate point. Similarly, image processing here is also processed using separate threads.

The workflow of the whole positioning system can be shown in Figure 7.

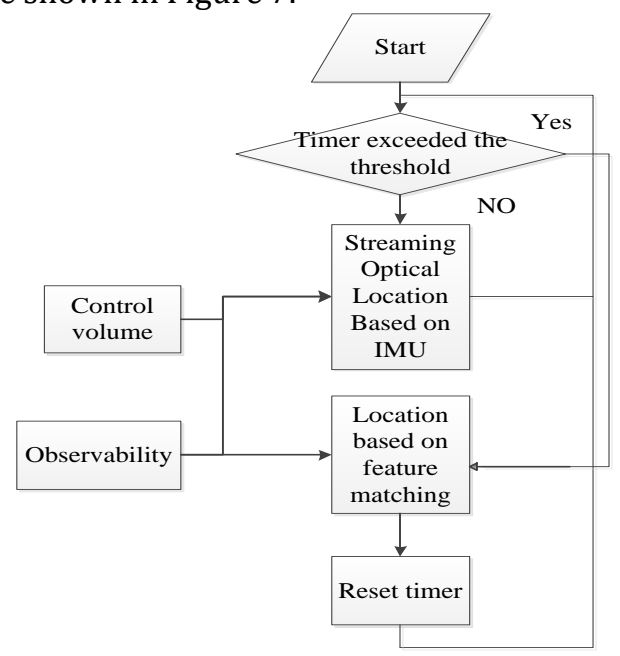

Figure 7: Workflow of positioning system

On the basis of the design of hardware circuit and software of the system, the detection and positioning system of low, slow, and small targets is tested, the measurement of the distance, height, speed and coordinate position of small targets is completed, and the error analysis of the test results is carried out.

In the experiment, DJI Elf 3 is selected as the low, slow, and small target to test the results. Meanwhile, the distance and height of the target, and the target velocity is measured by Doctor Energy 202540Elite1Mile CONX Laser Range Finder. The measured data are divided into two parts: reference value and test.

The first part uses Doctor Energy 202540Elite1Mile CONX Laser Range Finder to determine the position points of three known coordinates in the air. The origin of the coordinate system is the position of the observer.

The coordinate data of the three known points are used as reference data and compared with the subsequent coordinate data. 
The second part is the test. The DJI Elf 3 flies over the three known positions in turn. At the same time, the system designed by this subject is used to detect and locate the low, slow, and small targets.

The speed of the low, slow, and small targets is measured by CONX Multifunction Laser Range Finder. The test data are recorded and compared with the known reference data.

The working principle of the system is that the laser ranging sensor is fused in the GPS positioning module of the UAV, and the longitude and latitude of the machine are obtained by the GPS module. The distance from the machine to the target is measured by the laser ranging module, the angle between the line segment between the machine and the target and the direction of the magnetic north pole is obtained by the electronic compass, and then the pitch and roll angles between the machine and the horizontal plane is measured by the inclination measuring module. According to the measured data, the longitude and latitude of the target can be obtained by fusing them.

Laser ranging module: The module of this system adopts the laser ranging sensor designed in Chapter 3 above. The measurement range can reach $250 \mathrm{~m}$, and the measurement error is about $8.5 \mathrm{~cm}$. It emits laser pulses to the target to be measured, and receives the laser pulses reflected back by the target. By measuring the propagation time of the pulses, the distance between the UAV and the target is obtained, and then the data is sent to the data fusion module.

GPS positioning module: Receive GPS signals, determine the position of UAV, and send the position coordinates to data fusion module.

The GPS positioning module consists of two parts: PCB (Printed Circuit Board) board embedded with GPS chip and GPS antenna. GPS antenna is used to receive GPS signals, including coordinate information, UTC time, mode indication and other information.

PCB can use SIRFII single chip GSC3F/LP: the frequency of the chip is $\mathrm{L} 1,1575.42 \mathrm{MHz}$; the $\mathrm{C} / \mathrm{A}$ code is $1.023 \mathrm{MHz}$; the positioning accuracy is $2.5 \mathrm{~m}$, supporting serial communication. The differential positioning method can be used to obtain longitude and latitude information, thereby reducing errors. And positioning accuracy can reach centimeter level, in line with the requirements of the objectives.

The inclination module is used to measure the pitch and roll angles between the machine and the horizontal plane. Inclination module can choose ADXL345. ADXL345 is a three-axis acceleration measurement module, which is very suitable for mobile devices such as laser range finder and positioner, and can be used as a tilt sensor. It has low power consumption, high resolution (13 bits) and a measurement range of $\pm 16 \mathrm{~g}$. It can measure the static gravity acceleration in the application of inclination detection. By measuring the acceleration components on $\mathrm{X}$ axis and $\mathrm{Y}$ axis, the pitch angle and roll angle can be obtained, and the inclination change of 0.1 degree can be measured. ADXL345 uses a polycrystalline silicon surface micro-fabricated structure. The differential capacitor consists of an independent fixed plate and a movable mass connecting plate, which can measure the structural deflection. Acceleration can deflect inertia mass and unbalance differential capacitance, so the output amplitude of sensor is proportional to acceleration. Phase demodulation is used to determine the magnitude and polarity of acceleration.

Electronic compass module: used to measure the azimuth of the line between the machine and the target and the direction of the magnetic north pole. The electronic compass module can use Honeywell HMC5883L. It has a high cost performance ratio in the field of compass and magnetic field detection, and is widely used in consumer electronic equipment, such as mobile phones, tablet computers, navigation equipment, etc.

The Honeywell anisotropic magnetoresistance technology adopted by HMC5883L is obviously superior to other magnetic sensor technologies. It has strong anti-interference ability, stable temperature characteristics, wide operating frequency, high resolution, small size and easy installation. The solid phase structure of the sensor, which is low sensitive to the orthogonal axis, can be used to measure the direction and magnitude of the earth's magnetic field, ranging from $5 \mathrm{mG}$ to $8 \mathrm{mG}$. In addition, it has high integration and abundant builtin functions, including position/reset circuit, amplifier circuit, compensation circuit, filter circuit, and ADC circuit. It can realize automatic demagnetization, self-measurement and offset compensation, and can continuously output azimuth information.

Data fusion module: Data is processed by MCU (Micro Control Unit) and fused.

\section{Conclusion}

In recent years, the rapid growth of low-speed and small-target development trend has brought profound changes to all walks of life, but also caused many threats, such as interference with air routes, spying on key confidential areas, illegal use of criminal activities etc. Therefore, intrusion detection and effective interception of low, slow, and small targets have become a hot research topic in various countries. A method of track measurement and positioning for low, slow, and small targets using laser detection technology is proposed on the basis of a large number of literature inquiries and analysis of low, slow, and small target intrusion detection technology at home and abroad. Compared with radar technology, acoustic detection and radio scanning, this method has the characteristics of high efficiency and easy realization, and is more suitable for detecting and locating low-speed flying small targets in ultra-low altitude visual range.

The purpose is to design a laser ranging sensor with small size, low power consumption, and light weight. It combines with GPS sensor system, carries on the measurement of the target on the unmanned aerial vehicle, obtains the three-dimensional 
coordinates of the target surface, and then gets the volume of the target through data fitting and integration.

The main achievements are as follows:

The pulse laser ranging sensor and phase laser ranging sensor are analyzed in detail. Based on the design requirements, the pulse laser ranging sensor is selected and the simple structure of the laser ranging system block diagram is designed. At the same time, the measuring range and ranging accuracy of the pulse laser ranging system are analyzed.

According to the requirement of measuring range and accuracy, and the characteristics of low power consumption and small volume, the laser ranging system is designed and the components are selected, including transmitting module, receiving module, and time measurement module.

The UAV and GPS positioning and sensing system are studied and analyzed. Combining GPS positioning system with laser ranging system, the position of UAV is obtained according to GPS module, the distance between UAV and target is measured by laser ranging module, and the three-dimensional coordinates of the surface points of target object are obtained by data fusion.

By using the method of surface reconstruction, a series of discrete points of three-dimensional coordinates on the surface of the target object are obtained for surface fitting, and the volume of the three-dimensional object is calculated.

In summary, although the low, slow, and small target detection and positioning system designed has realized the basic positioning function, there are still some deficiencies in the design due to the more content involved in the system and the limitation of time and level.

Firstly, the influence of temperature on the circuit performance is not considered in the design. Especially the photodetector APD should try to design a good temperature control compensation circuit to reduce the influence of temperature on the detection efficiency.

Second, in the time discrimination circuit, RC delay circuit with simple structure is used in the design of signal delay circuit. Although it is easy to realize, the delay precision of signal is not high, and the waveform of signal is easy to be distorted. Further research should consider the use of highprecision dedicated delay chip.

Third, only the circuit design part is studied, but the optical system has not been studied in depth. The next step is to design a good optical system to match the circuit part, so as to further improve the detection and positioning accuracy.

Moreover, this subject only carries out some laboratory theoretical research, and there is still a long way to go from the specific application.

\section{Acknowledgement}

Research startup subject of Yangtze Normal Universi ty: 2017KYQD16

\section{References}

[1] Guerra, E., Munguía, R., \& Grau, A. (2018). Uav visual and laser sensors fusion for detection and positioning in industrial applications. Sensors, 18(7), 738.

[2] Gallay, M., Eck, C., Zgraggen, C., Kaňuk, J., \& Dvorný, E. (2016). High resolution airborne laser scanning and hyperspectral imaging with a small uav platform. ISPRS - International Archives of the Photogrammetry, Remote Sensing and Spatial Information Sciences, XLI-B1, 823-827.

[3] Lu, Y., Xue, Z., Xia, G. S., \& Zhang, L. (2018). A survey on vision-based uav navigation. Geospatial Information Science, 1-12.

[4] Sun, R., Cheng, Q., Wang, G., \& Ochieng, W. Y. (2017). A novel online data-driven algorithm for detecting uav navigation sensor faults: Sensors, 17(10), 2243-.

[5] Liu, X. J., Tao, X. M., Duan, Y. P., \& Ge, N. (2017). Visual information assisted uav positioning using priori remote-sensing information. Multimedia Tools \& Applications, (6), 1-20.

[6] Mac, T. T., Copot, C., Keyser, R. D., \& Ionescu, C. M. (2018). The development of an autonomous navigation system with optimal control of an uav in partly unknown indoor environment. Mechatronics, 49, 187-196.

[7] Bu, J., Sun, R., Bai, H., Xu, R., Xie, F., \& Zhang, Y., et al. (2017). Integrated method for the uav navigation sensor anomaly detection. Iet Radar Sonar \& Navigation, 11(5), 847-853.

[8] He, S., Xue, Y., Lin, J., Zhang, L., Du, X., \& Chen, L. (2016). Effect of silane coupling agent on the structure and mechanical properties of nano-dispersed clay filled styrene butadiene rubber. Polymer Composites, 37(3), 890-896.

[9] Vetrella, A. R., Fasano, G., Accardo, D., \& Moccia, A. (2016). Differential gnss and vision-based tracking to improve navigation performance in cooperative multi-uav systems. Sensors, 16(12), 2164-.

[10] Piotr, K., Rafał, G., \& Stanisław, K. (2017). Estimation of uav position with use of smoothing algorithms. Metrology \& Measurement Systems, 24(1).

[11] Nguyen, P. H., Arsalan, M., Koo, J. H., Naqvi, R. A., Truong, N. Q., \& Park, K. R. (2018). Lightdenseyolo: a fast and accurate marker tracker for autonomous uav landing by visible light camera sensor on drone. Sensors, 18(6), 1315.

[12] Jin, Z., Wang, X., Moran, B., \& Quan, P. C. Z. (2016). Multi-region scene matching based localisation for autonomous vision navigation of uavs. Journal of Navigation, 69(6), 1215-1233. 